\title{
Design and Implementation of Data Gathering Application Software Based on Android Mobile Terminal
}

\author{
Ran Li, Li Lou, Yong-Yang Hu \\ Nanjing Telecommunication Technology Institute, \\ Jiangsu, China \\ E-mail: Ryanlee730@163.com, louie1000@outlook.com
}

\begin{abstract}
In the data gathering area, mobile terminals with various sensors and abundant user interfaces can provide functions of data gathering, storage and transport on time. Meanwhile, the intelligent OS, e.g., Android, which has properties like open-source, equally extended applications, strong compatibility, etc., is suitable for application software development on mobile terminals. In this paper, a data gathering application software running on mobile terminal based on Android is designed and implemented. The design scheme of software architecture, data gathering, storage flow, and the implement of main functions of the application are also introduced.
\end{abstract}

Keywords-android; mobile terminal; data gathering software

\section{INTRODUCTION}

Mobile terminal or mobile communication terminal is a computer device that can be applied while moving. In most cases, mobile terminals have various functions like intelligence cell phone and tablet computer. Mobile terminal is usually equipped with abundant process capability, memory, storage and operating system to handle comparatively complex tasks. Relevant techniques of mobile terminal include chip, system software, interactive model between human and computer, technique of application development, etc. Specifically, arm embedded processor with low power consumption is dominating in the mobile terminals and open-source OS is the main development direction of the mobile terminal operating system.

Android is an open-sourced operating system released by Google based on Linux [1], which is consist of operating system, middleware, user interface and application software. Nowadays, Android is mainly applied in mobile device like intelligent cell phone, tablet computer, etc. Android is first developed by Andy Rubin, and mainly used for supporting cell phone. In 2005, Android is purchased and financed by Google. In November of 2007, Open Handset Alliance is founded by Google and 84 hardware manufacturer, software developer and telecom operators, who are responsible for developing and improving Android, and its source code is released through apache open source license warrant by Google. The first intelligent cell phone with Android was released in October 2008, and then was extended to tablet computer and other areas. Compare with other data gathering applications [2-5], Android fits well for mobile terminal application development due to its open source, equally extended application and strong compatibility.

In this paper, a device data gathering software is implemented based on intelligent operation system Android and mobile terminal. The design scheme of software architecture, data gathering, storage flow, and the implement of main functions of the software are also introduced. The rest of paper is structured as follows. Section II gives the general architecture of Android applications and main constitute elements. The design scheme of the system architecture, data gathering and storage flow is introduced in section III. In section IV, the implementation for main function of software is introduced. Section $\mathrm{V}$ makes conclusion and gives the future work.

\section{ARCHITECTURE OF ANDROID APPLICATIONS}

Android application includes low-level operating system, middleware and high-level applications. As shown in Figure 1, the architecture of Android applications includes 4 levels from low to high: operating system level, system running library level, application framework level and application level. 


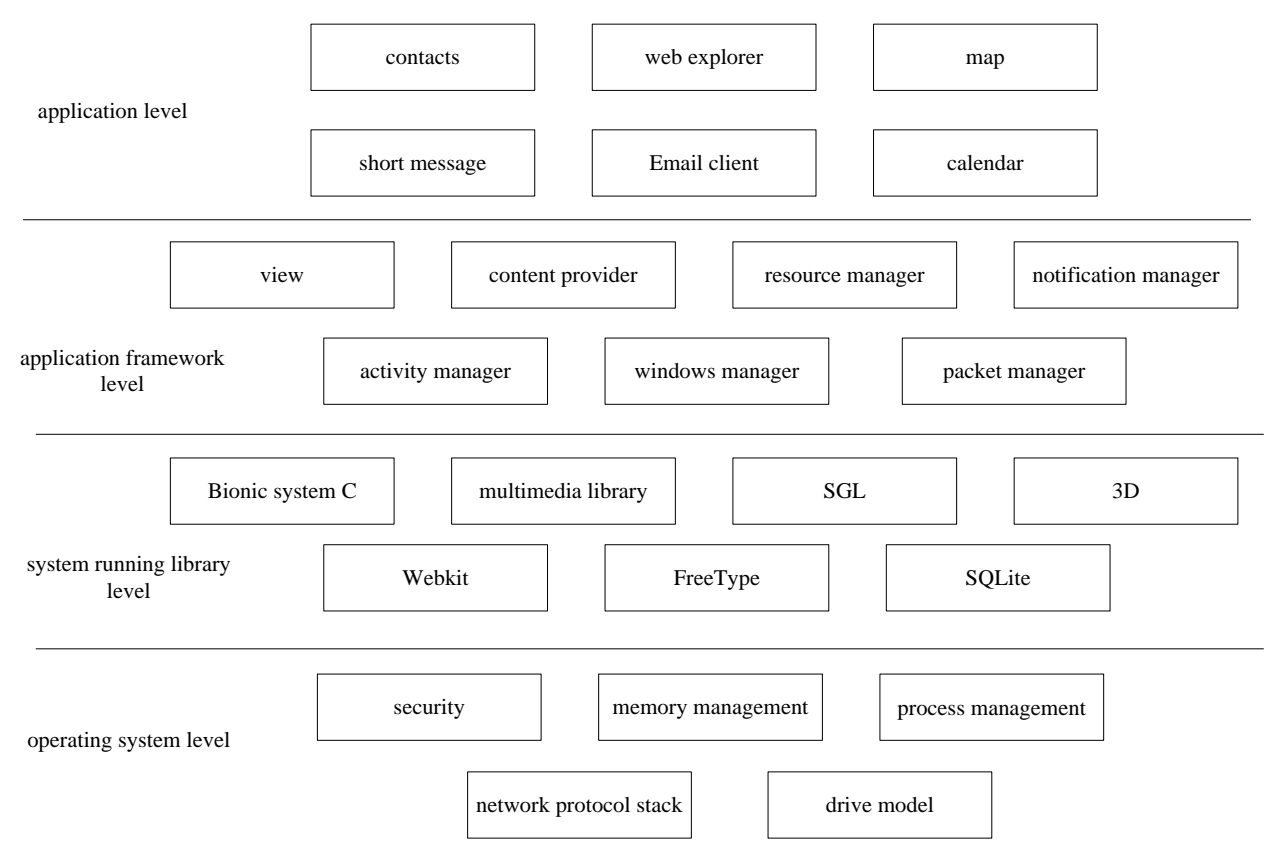

Figure 1. Architecture of Android application.

Operating system level: the core service of operating system in operating system level is based on Linux2.6, which provides low-level service of system including security, memory management, process management, network protocol stack, drive model, etc.

System running library level: system running library level include $\mathrm{C} / \mathrm{C}++$ libraries provided for Android components and Android running library, which including Bionic system C library, multimedia library, 2D image engine library SGL, 3D effects support library OpenGL, bitmap and vector library FreeType, relational database SQLite, web explorer engine Webkit, etc.

Application framework level: application framework level provide high level application API framework for developers, including view, content provider, resource manager, notification manager, activity manager, windows manager, package manager, etc.

Application level: application level include program running on virtual machine coded in Java and high level core application program package, such as contacts, web explorer, map, calendar, short message, Email client, etc.

Android application is programmed by Java language, which includes resource file in res directory, program file in src directory and APK package complied through resource files. Application programmed on Android is composed of components coupled loosely, and there is a manifest file to describe interaction in a single component or between different components. Android application is composed by 6 basic application components as follows $[1,6]$ :

Activities: Each Android application interface is extended by basic activity class. Activity use view to construct user interface for displaying information and responding users' operating. For desktop application, an activity is corresponding to a window.
Intent: Intent is framework for passing message between applications, which implements switch between different activities.

Service: Service is running backstage and its main function is triggering notification when updating data source and visible activity. Service keeps applications running when activity is becoming invisible and inactive.

Content Provider: Content provider is responsible for sharing data storage, implement data sharing and access between applications. Content provider provides a series of criterion and method for other applications to save and read data type provided.

Broadcast Receivers: Broadcast Receivers open application and response receiving intent. Application listens and matches broadcast intent with specific filer criterion through creating and registering broadcast receivers.

Notifications: Notifications notify users without interrupting current activity or focus.

\section{DESIGN SCHEME OF DATA GATHERING SYSTEM SOFTWARE}

The design of data gathering system software include system architecture, data gather flow and data storage.

\section{A. System Architecture Design}

The design of system architecture is shown in Figure 2, which can be divided into sensing level, network level and application level [7,8]. Sensing level include mobile terminal based on Android, which is applied for gathering data. Network level includes wireless communication network, sensor network and Ethernet where TCP/IP [9] is adopted. It provides universe software interface for topper level through middleware. Application level include main function of system including data gathering, data displaying, timing and locating, etc. 


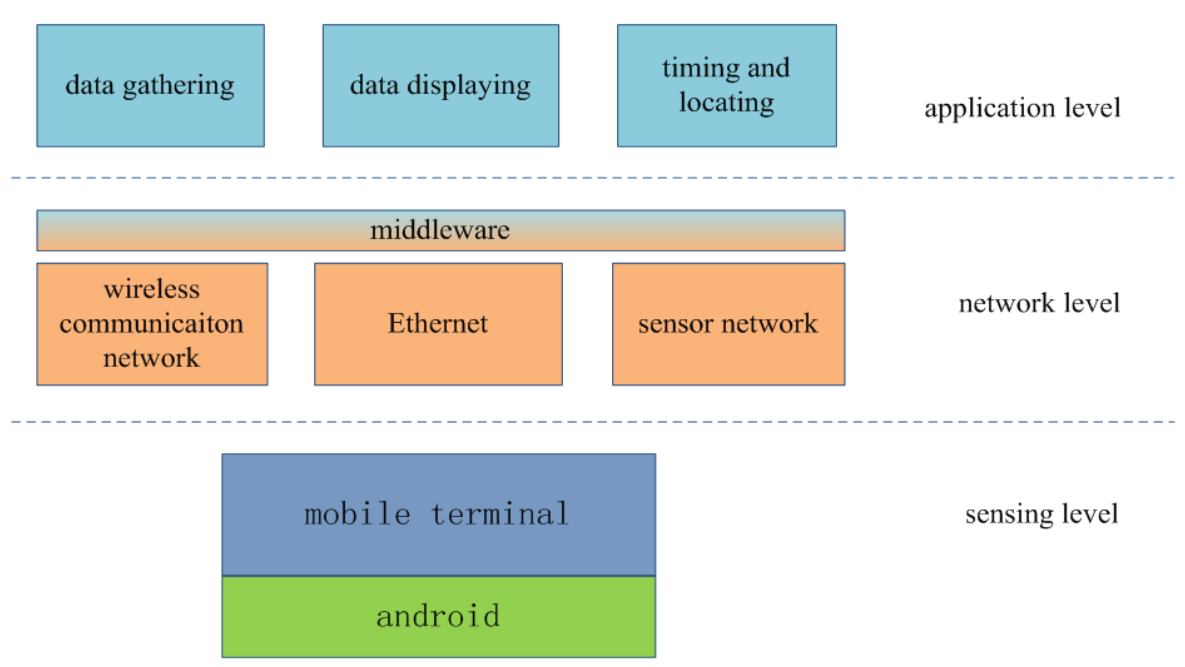

Figure 2. System architecture.

Data gathering software architecture is shown in Figure 3, which includes 3 main components: timing and locating component, data gathering component, data processing and storage component. Data gathering software provide friendly interface displaying on terminal for data gathering people, which have functions of automatic timing and locating, data gathering as well as data processing and storage on real time. The data gathered through terminal software can input into PC server both on-line and off-line.

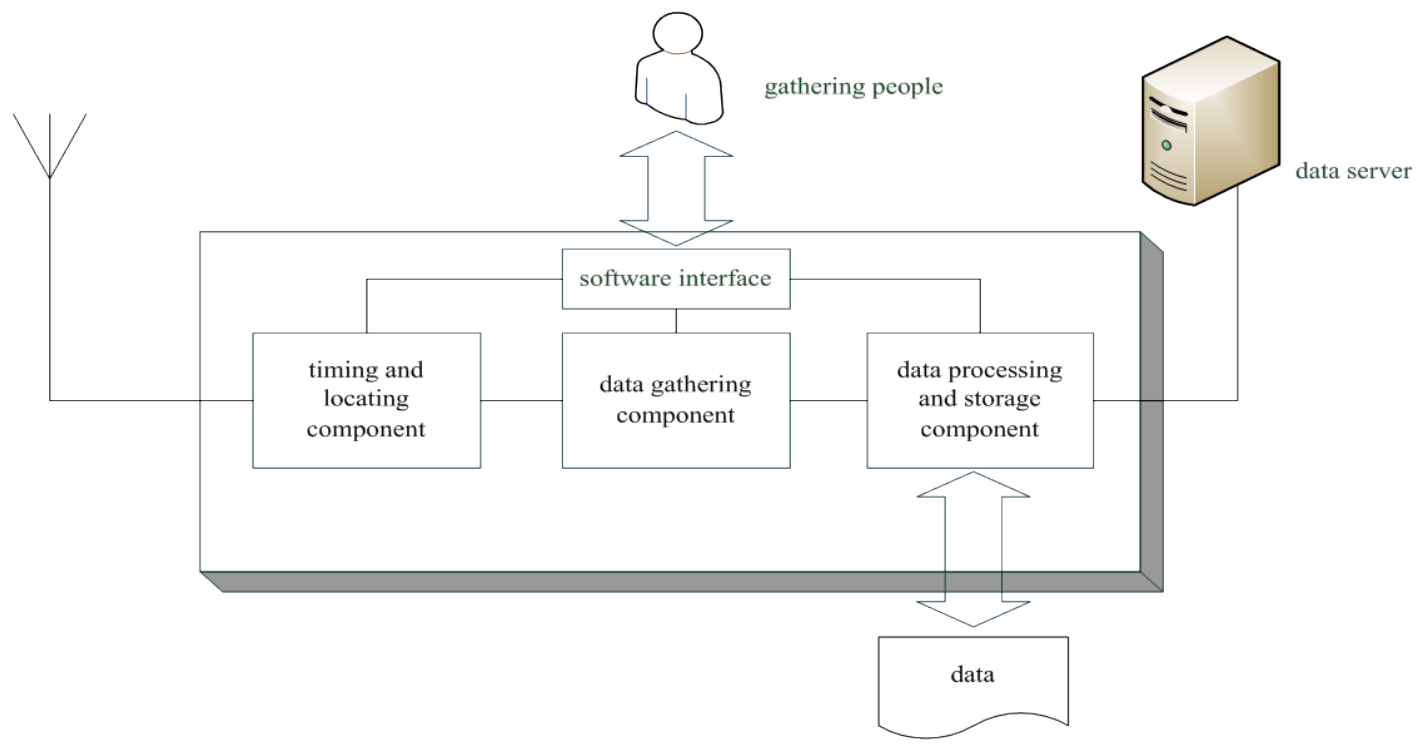

Figure 3. Software architecture of data gathering software.

\section{B. Design of Data Gathering Flow}

Based on various UI controls, in the application software, there are 3 kinds of interface to response users' event, including user login interface, device select interface and device data interface, corresponding to 3 kinds of activity in Android applications. Figure 4 is the flow of data gathering in software. User login in interface is loaded and displayed firstly when the application is start, and user login in the system through user name and password; application switch to device selection interface once validation process is passed, waiting for the device is confirmed by user. Then the relevant interface of device data is loaded and displayed for data gathering. When all data is gathered, integrity of data will be inspected by the software, if integrity is not satisfied, the data form will return to user for further data gathering; in the end, data is stored by application to finish the process of data gathering. 


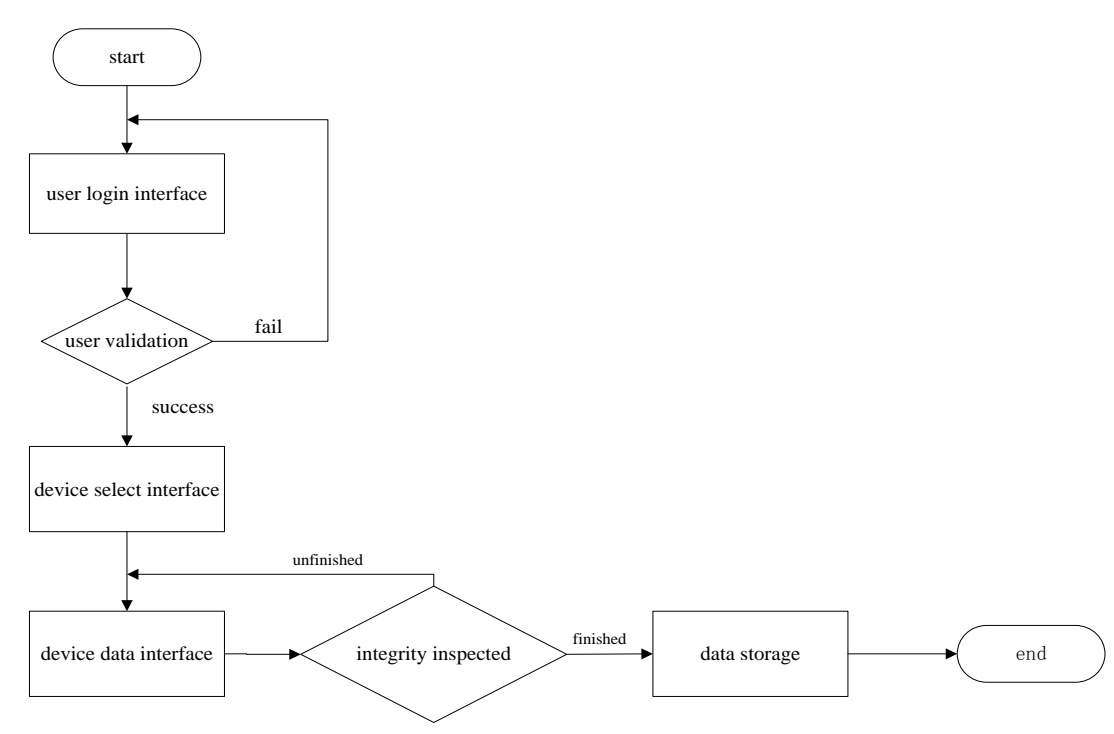

Figure 4. Flow of data gathering.

\section{Design of Data Storage of Software}

Currently, there are 3 main data storage methods in Android applications: Sharedpreferenc storage, file storage and SQLite database storage. The compare of 3 storage methods in characteristic, applied scope and implementation complexity is shown in Table 1.

TABLE I. TABLE STORAGE METHOD IN ANDROID APPLICATIONS

\begin{tabular}{|c|c|c|c|}
\hline & introduction & applied scope & complexity \\
\hline sharedpreference & $\begin{array}{c}\text { light storage on } \\
\text { Android; store } \\
\text { and query by key- } \\
\text { pairs }\end{array}$ & $\begin{array}{c}\text { small amount of } \\
\text { structured data } \\
\text { storage; simple } \\
\text { key pair data }\end{array}$ & \\
\hline file storage & $\begin{array}{c}\text { data read, write } \\
\text { and store through } \\
\text { file stream }\end{array}$ & $\begin{array}{c}\text { text and } \\
\text { multimedia } \\
\text { resource storage }\end{array}$ & medium \\
\hline $\begin{array}{c}\text { SQLite database } \\
\text { storage }\end{array}$ & $\begin{array}{c}\text { apply relational } \\
\text { database; store } \\
\text { data with table }\end{array}$ & $\begin{array}{c}\text { complicated } \\
\text { structured data } \\
\text { storage, insert } \\
\text { and query }\end{array}$ & \\
& structure; & complicated \\
& operating & database & \\
& database through & frequently & \\
& SQL & & \\
\hline
\end{tabular}

Consider the data property gathered in software, file storage is selected in our application. Figure 5 is the flow of data storage in the software based on SD card in the terminal. Firstly, the authority to access SD card is obtained by the application and a XML file is created in the SD card. Then data tab is created and relevant data value is written in the XML file based on data type gathered. Finally, XML file is closed and data store process is finished.

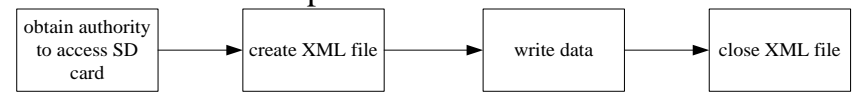

Figure 5. Flow of data storage based on SD card.

\section{IMPLEMENTATION OF MAIN FUNCTION OF DATA GATHERING SOFTWARE}

\section{A. Android Mobile Terminal and Application Development Environment}

The implementation of data gathering system is based on intelligent information terminal. The main hardware components of mobile terminal are shown in Figure 6. The hardware of terminal includes core process unit, timing and locating unit, power supply unit, etc. Information terminal integrates locating antenna, display screen, touch screen, function button, lithium battery, etc. The interface of Ethernet provides function of $100 \mathrm{M}$ communication, which can connect into Ethernet to implement communications between terminal and PC servers [10]. Interface of memory card can install large capacity memory card for storing map and software data. Power interface can provide power for terminal and charge power for inner lithium battery. Audio interface provide interface for audio input and output.

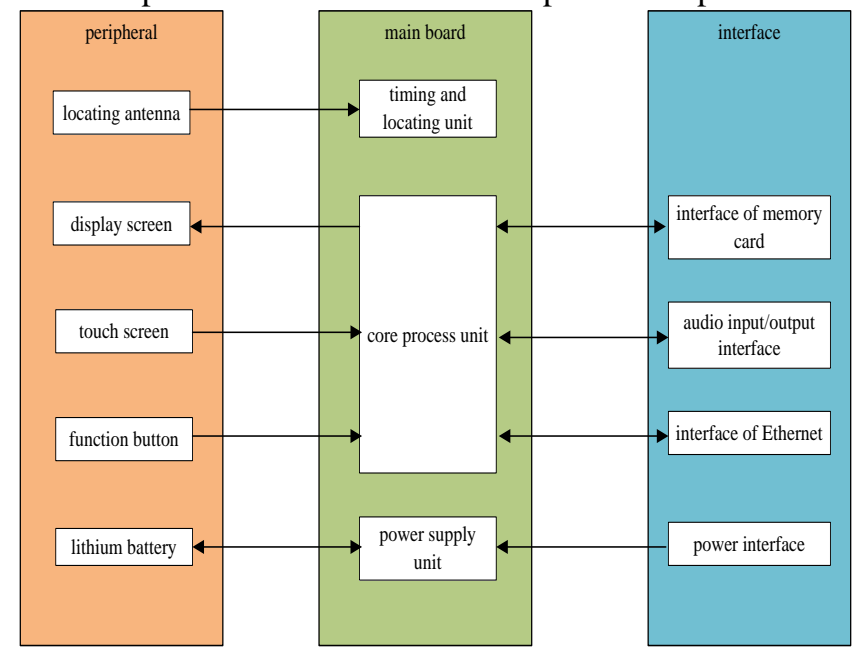

Figure 6. Hardware components of mobile terminal. 
The main hardware parameters of mobile terminal are shown in Table 2. The applied terminal is equipped with $1 \mathrm{GHz}$ arm CPU, 512M DDR memory, supporting Ethernet, GPS, USB, etc.

TABLE II. TABLE HARDWARE PARAMETER OF ANDROID MOBILE TERMINAL

\begin{tabular}{|c|c|}
\hline hardware & parameter \\
\hline CPU & 1GHz ARM processor \\
\hline Memory & DDR 512MB \\
\hline Rom & TF card 32GB max \\
\hline Display screen & 5 inches capacitive touch screen \\
& $800 * 480$ resolution \\
\hline Operating system & Android 4.0 \\
\hline Networks & Wireless network; 100M Ethernet; GPS \\
\hline
\end{tabular}

The software of data gathering is developed in Eclipse, which is an open-source and cross-platform integrate development environment. The project directory structure is shown in Figure 7, where resource file is in res directory, including XML configure files, image, UI layout files, etc.; java source code is in src directory and is organized according java package rules; files generated by Android development tools are in gen directory, where R.java is auto updated by development tools, including the property of all the files in res directory; AndroidManifest.xml is declaration file of Android application, including important information required by Android program as application name, icon, package name, modules, etc. [11-13].

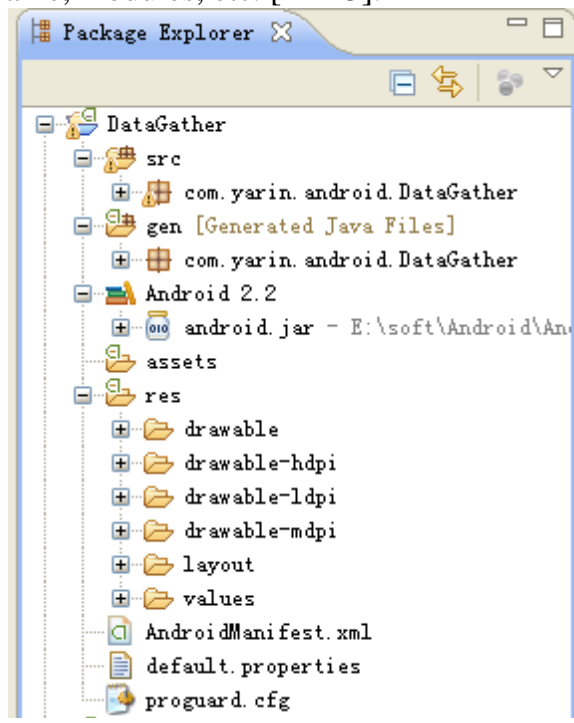

Figure 7. Project directory structure of software.

\section{B. Implementation of Software Interface}

Activity is a basic module in Android applications. An activity is corresponding to a single form or screen in terminal and is implemented by a java class, which is inherited from basic activity class. User interface is composed of various controls for responding events. During the development process of Android software interface, the layout of interface should be set in res/layout directory of the project firstly, where a layout file corresponding to an interface in future application. Our data gathering application applied multiple controls for gathering device data, such as TextView, EditText, RadioButton, CheckBox, Spinner, etc. The interface layout is set and modified by layout xml files.

Form of device contains basic information of device such as device name and version. Due to the limitation of terminal screen width, a series of tab page is designed to display relevant contents, such as basic information, performance data, reactive data, motorized data, etc. The software sets the layout of interface in layout files and adds each Tab page into the TabActivity class.

\section{Auto Obtain of Date, Time, Altitude, Longitude and Latitude}

The date and time of software are automatically obtained based on system time; altitude, longitude and latitude of software are obtained by GPS module of mobile terminal. All the obtained data will be filled automatically into the relevant position of the software interface for users.

Obtain process of date and time is based on instantiated calendar class of Android, after form conversion of date and time, they will be displayed in a textbox control on the screen. The implementation flow of date and time automatic obtain is shown is Figure 8.

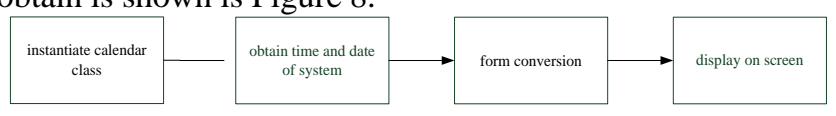

Figure 8. Flow of date and time automatic obtain.

Obtain process of altitude, longitude and latitude is based on GPS module equipped in the terminal, after get the system service of positioning and set a listener, the position information is obtained through a function for responding position changed, and then displayed on the screen. The implementation flow of altitude, longitude and latitude automatic obtain is shown is in Figure 9.

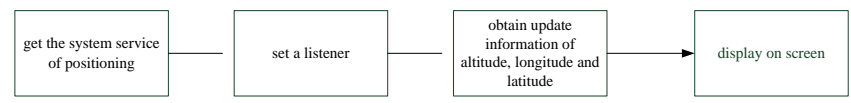

Figure 9. Flow of altitude, longitude and latitude automatic obtain.

\section{Implementation of Data Storage}

The process for implementation of system data storage firstly adds authority of SD card access in AndroidManifest.xml of project:

$<$ !--AndroidManifest.xml-->

$<!$ - authority for creating and deleting files in SD card--> $<$ uses-permission

Android:name="Android.permission.MOUNT_UNMOUNT _FILESYSYTEM">

$</$ uses-permission $>$

$<$ !-- authority for writing data into files in SD card -- $>$

$<$ uses-permission

Android:name="Android.permission.WRITE_EXTERNAL_ STORAGE">

$<$ /uses-permission>

Then, a button for gather finished is added in the interface and a listening event is added for the button. A file output stream is defined to write the relevant tab into a XML 
file, which will be closed after data gathering. The flow of data storage is shown in Figure 10.

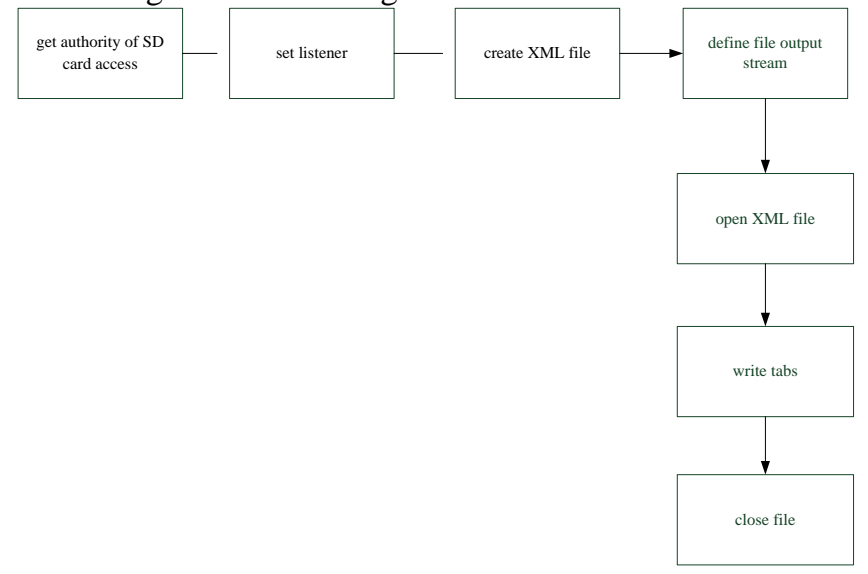

Figure 10. Flow of data storage.

\section{CONCLUSION AND FUTURE WORKS}

In the data gathering area, mobile terminals with various sensors and abundant user interfaces can provide functions of data gathering, storage and transport on time. Leveraged by the intelligent operating system, e.g., Android, an application conducting data gathering and storage is proposed and implemented in this paper. Specifically, the main design schemes as well as the detailed implementation methods are introduced. As a successive work, optimized methods of the data analyzing and processing will be studied in the future.

\section{ACKNOWLEDGMENT}

This work was supported by National Natural Science Foundation of China under Grant No. 61402521 and No. 61201216, Jiangsu Province Natural Science Foundation of China under Grant No. BK20140068.

\section{REFERENCES}

[1] Code Home. Android-An Open Handset Alliance Project [EB/OL].http://code.google.com/Android/what-is-Android html,2008.

[2] W. Souza, K. Okada. Mobile data collection. User Experience Magazine, 2012, 11(2).

[3] Microsoft Device Team. Nokia data gathering: a mobile tool with social impact. https://blogs.windows.com/devices/2012/07/19/nokiadata-gathering-a-mobile-tool-with-socialimpact/\#iEqPyicStULlFcRO.97

[4] Microsoft Data Gathering. Offers organizations a fast, accurate, cost effective and user-friendly way to collect data using mobile devices. https://www.microsoftdatagathering.net.

[5] M. Paschou, E. Sakkopoulos, A. Tsakalidis. Easy HealthApps: eHealth Apps dynamic generation for smartphones and tablets [J]. Journal of Medical Systems, 2013, 37(3): 1-12.

[6] Androlib. Number of new applcations in Android market by month, 2012. http://www.androlib.com/appstats.aspx.

[7] Anup Kale, Zenon Chaczko, Shaher Slehat. HyMuDs: A Hybrid Multimodal Data Acquisition System [C]. 2015 Asia-Pacific Conference on Computer Aided System Engineering. 2015 IEEE. 107-112.

[8] Quan Xu,Jiucheng Liu,Lei Zhang. A Lightweight Framework for Data Acquisition and Quality Monitoring in Power System [C]. Proceeding of the 11th World Congress on Intelligent Control and Automation.2014 IEEE: 2956-2960.

[9] G. Wang, Y. Ren, and J. Li. An effective approach to alleviating the challenges of transmission control protocol [J]. IET Communications, 2014, 8(6): 860-869.

[10] G. Wang, Y. Wu, K. Dou, et al. AppTCP: The design and evaluation of application-based TCP for e-VLBI in fast long distance networks. Future Generation Computer Systems, vol. 39, pp. 67-74, 2014.

[11] A.P.Felt, E.Ha, S.Egelman, et al. Android Permissions: User Attention,Comprehension and Behavior [R]. Uniersity of California,Berkeley,2012.

[12] A. P. Felt, H. Wang, A. Moshchuk, et al. Permission Re-Delegation: attacks and defenses. In USENIX Security Symposium, 2011.

[13] A.P. Felt, E. Chin, S. Hanna, et al. Android permissions demystified. In ACM CCS, 2011. 